\title{
Disease-related malnutrition but not underweight by BMI is reflected by disturbed electric tissue properties in the bioelectrical impedance vector analysis
}

\author{
Kristina Norman*, Christine Smoliner, Anne Kilbert, Luzia Valentini, Herbert Lochs and Matthias Pirlich \\ Charite Universitätsmedizin, Klinik für Gastroenterologie, Hepatologie und Endokrinologie, Charitéplatz, 1, Berlin 10117, \\ Germany
}

(Received 27 September 2007 - Revised 6 December 2007 - Accepted 10 December 2007 - First published online 31 January 2008)

The calculation of body composition using bioelectrical impedance analysis in sick and hospitalized patients is hampered due to altered hydration state. We wanted to investigate how disease-related malnutrition assessed by the Subjective Global Assessment (SGA) is reflected in the bioelectrical impedance vector analysis. Patients with benign gastrointestinal disease $(n 242)$ were entered in the study. Nutritional status was assessed by SGA. Arm muscle and fat area were estimated by anthropometry, muscle function was determined by hand grip strength. Whole body impedance measurements were made at $50 \mathrm{kHz}$. Ninety-eight patients were considered well nourished (SGA A), ninety-four were classified moderately malnourished (SGA B) and fifty patients were classified severely malnourished (SGA C) according to the SGA. The mean vector was significantly displaced between SGA C and SGA A and B, showing comparable resistance with a significantly reduced reactance, indicating comparable hydration but loss of dielectrical mass of soft tissues. This distinctive vector migration was not seen when studying the patients grouped according to BMI. In conclusion, disease-related malnutrition as assessed by the SGA is associated with a distinctive bioelectrical vector migration, implying that abnormal tissue structure and not reduced body mass only occurs in disease-related malnutrition. These disturbances are not seen in underweight according to BMI. Bioelectrical impedance vector analysis appears to be an attractive tool to identify disease-related malnutrition and to monitor nutritional intervention.

Disease-related malnutrition: Bioelectrical impedance vector analysis: Subjective Global Assessment: BMI: Phase angle: Gastrointestinal disease

Disease-related malnutrition is a common complication in sick and hospitalized patients with deleterious effects on morbidity and mortality ${ }^{(1)}$. Malnourished patients have an impaired muscle function, poorer functionality and experience a lower quality of life $e^{(2,3)}$.

Several studies have shown that malnourished patients exhibit lower body cell mass assessed either by bioelectrical impedance analysis (BIA) or more sophisticated methods ${ }^{(3,4)}$.

Assessing body composition in hospitalized patients is, however, not always easy, since sophisticated methods are time consuming, expensive and not suitable for routine clinical use. The non-invasive BIA is a simpler method which can easily be employed as a bed-side method in hospital. BIA is considered a reliable tool for body compartment calculation in healthy individuals who have no fluid imbalance, no body shape abnormalities and are within a certain BMI range $\left(16-34 \mathrm{~kg} / \mathrm{m}^{2}\right)$. Furthermore, appropriate (i.e. age-, sex- and population-specific) equations for the calculation of body compartments must be applied ${ }^{(5)}$.

However, these necessary assumptions for the calculation of body compartments using the impedance parameters obtained by BIA (e.g. homogenous composition, fixed cross-sectional area and consistent distribution of current density) are frequently not applicable in sick and hospitalized patients and this is mainly due to disturbed hydration as in nephrotic syndrome, renal failure, cardiac insufficiency, obesity or liver cirrhosis $^{(6-9)}$, or modifications in distribution of extra- and intracellular water.

Bioelectrical impedance vector analysis (BIVA), on the other hand, uses the plot of the impedance parameters resistance $(\mathrm{R})$ and reactance $(\mathrm{Xc})$ normalized per height as a bivariate vector in the $\mathrm{R}$ Xc $\operatorname{graph}^{(10)}$. BIVA thus provides information about hydration status and body cell mass and integrity since reactance is the capacitative effect produced by the tissue interfaces and cell membranes, whereas bioelectric resistance is the pure opposition of a biological conductor to the flow of an alternating electric current. It has gained attention since it has been shown to be clinically useful in monitoring hydration status in renal patients undergoing chronic haemodialysis ${ }^{(7)}$, patients with liver cirrhosis ${ }^{(11)}$, critically ill patients ${ }^{(12)}$ and obese patients with stable and changing weight ${ }^{(13)}$.

Abbreviations: BIA, bioelectrical impedance analysis; BIVA, bioelectrical impedance vector analysis; R, resistance; R/H, resistance standardized per height; SGA, Subjective Global Assessment; Xc, reactance; Xc/H, reactance standardized per height.

* Corresponding author: Dr Kristina Norman, fax + 4930450514 923, email kristina.norman@charite.de 
In the present observational study, we wanted to investigate how benign disease-related malnutrition assessed by the Subjective Global Assessment (SGA), as well as nutritional status by $\mathrm{BMI}$, are reflected in the $\mathrm{R} \mathrm{Xc}$ graph.

\section{Subjects and methods}

Patients ( $n$ 242) who were hospitalized at our Department of Gastroenterology, Hepatology and Endocrinology were recruited for the study. Patients were considered for inclusion if they were older than 18 years and were suffering from benign gastrointestinal disease.

A priori defined exclusion criteria were malignant disease and life expectancy less than three months. Severe hyperhydration, estimated by clinical assessment and by ultrasound in patients with liver cirrhosis, as well as implanted defibrillators were considered further exclusion criteria for the BIA.

All measurements were made within $48 \mathrm{~h}$ of admission to hospital. All patients gave written informed consent and the Ethics Committee of the Charite Universitätsmedizin approved the study.

\section{Subjective Global Assessment}

The SGA was carried out using the protocol developed by Detsky et al. ${ }^{(14)}$. It relies on the patient's history regarding weight loss, dietary intake, gastrointestinal symptoms, functional capacity and physical signs of malnutrition (loss of subcutaneous fat or muscle mass, oedema, ascites). Patients were classified as well nourished (A), moderately or suspected of being malnourished (B) or severely malnourished (C).

\section{Whole body impedance measurements}

BIA was performed using a Nutriguard M (Data Input GmbH, Darmstadt, Germany) applying alternating electric currents of $800 \mu \mathrm{A}$ at $50 \mathrm{kHz}$ and $\mathrm{R}$ and $\mathrm{Xc}$ were measured. The phase angle reflects the contributions between $\mathrm{R}$ and $\mathrm{Xc}$ (i.e. the arc tangent of the ratio of reactance to resistance transformed to degrees).

Patients were measured in the morning after an overnight fast, in the supine position with arms and legs abducted from the body. Source and sensor electrodes $(\mathrm{Ag} / \mathrm{AgCl}$, Bianostic Classic ${ }^{\circledR}$ Electrodes; Data Input) were placed on the dorsum of both hand and foot of the dominant side of the body.

The $\mathrm{CV}$ of repeated measurements of $\mathrm{R}$ and $\mathrm{Xc}$ at $50 \mathrm{kHz}$ was assessed in five patients: $\mathrm{CV}$ was $<1.5 \%$ for $\mathrm{R}$ and $<2.6 \%$ for Xc.

$R X c$ graph. BIVA uses the plot of direct measurements of the vector components $\mathrm{R}$ and $\mathrm{Xc}$. According to the $\mathrm{R} \mathrm{Xc}$ graph, $\mathrm{R}$ and $\mathrm{Xc}$ normalized for body height are plotted as a bivariate random vector $(\mathrm{Xc} / \mathrm{H} v . \mathrm{R} / \mathrm{H})$ on the $\mathrm{R}-\mathrm{Xc}$ plane. The vector distribution is described by its associated $95 \%$ CI (confidence ellipse in the $\mathrm{R}-\mathrm{Xc}$ plane). The shortening or lengthening of the vector indicates hydration status in the form of oedema or dehydration, respectively, whereas a migration sideways indicates increase or decrease in dielectric mass (cell membranes and tissue interfaces) of soft tissues ${ }^{(10)}$.

\section{Anthropometric measurements}

Weight and height were documented and used to calculate BMI (weight $(\mathrm{kg}) /$ height $\left.(\mathrm{m})^{2}\right)$. Mid upper arm circumference was determined and triceps skinfold was measured with a Holtain caliper (Crymych, UK) on the non-dominant arm. Arm muscle area and arm fat area were calculated applying the formula by Gurney \& Jelliffe ${ }^{(15)}$.

\section{Muscle function}

Handgrip strength was measured in the non-dominant hand with a Jamar ${ }^{\circledR}$ dynamometer. The patients performed the test while sitting comfortably with shoulder adducted and neutrally rotated, the elbow flexed to $90^{\circ}$, forearm and wrist in neutral position. The patients were instructed to perform a maximal isometric contraction. The test was repeated twice within $30 \mathrm{~s}$ and the highest value of the three measurements was recorded.

\section{Laboratory parameter}

All laboratory parameters (C-reactive protein, albumin) were determined according to standard methods.

\section{Statistics}

Statistical analysis was carried out using the software package SPSS $^{\odot}$ version 13 (SPSS Inc., Chicago, IL, USA).

All data are presented as median and interquartile range. Multiple comparison was performed by Kruskal-Wallis test. Following a significant test result, comparisons between the SGA classes or BMI classes were performed using the Mann-Whitney $U$ test. Spearman's correlation was calculated to assess the relationship between variables.

Statistically significant differences between the mean vectors of the SGA or BMI classes were assessed with the Hotelling's $T^{2}$ test for vector analysis. Mahalanobis distance $D$, a generalized measure of distance between the groups defined by two correlated variables, was also calculated. Vector analysis was performed with BIVA software ${ }^{(16)}$.

An acceptable level of statistical significance was established $a$ priori at $P<0 \cdot 05$.

\section{Results}

In total 242 patients (121 female) were recruited for the study. All patients suffered from benign gastrointestinal disease, mostly liver disease ( $n$ 82), chronic inflammatory bowel disease ( $n$ 82), biliary ( $n$ 16), gastritis/oesophagitis ( $n$ 22), pancreatic disease ( $n$ 13), diverticulosis/diverticulitis $(n$ 6) and other $(n 21)$. Ethnic origin of all patients was Caucasian.

\section{Disease-related malnutrition}

Ninety-eight patients were considered well nourished (40\%), ninety-four were classified moderately malnourished (39\%) and fifty patients were classified severely malnourished $(21 \%)$ according to the SGA.

Age was not significantly different between well-nourished, moderately and severely malnourished patients and sex 
distribution was only different between well-nourished and severely malnourished with a higher percentage of men in the SGA class $\mathrm{C}$ as shown in Table 1. BMI, arm muscle area and arm fat area decreased significantly with SGA class and albumin and C-reactive protein was significantly different only between well-nourished and moderately as well as severely malnourished. Hand grip strength was significantly lower in severely malnourished than moderately malnourished or well-nourished patients.

\section{Impedance parameters}

Phase angle decreased significantly with SGA class (see Fig. 1). We also obtained a discreet correlation between percentage weight change in the preceding 6 months and the phase angle $(r \quad 0.221, P=0.001)$, and with $\mathrm{R} / \mathrm{H}$ at $50 \mathrm{kHz}$ $(r-0.203, P=0.002)$.

A significant vector displacement was observed between moderately and severely malnourished patients due to a reduced Xc component with preserved $\mathrm{R}$, whereas there was no significant difference between the mean vector position of well-nourished and moderately malnourished patients (see Fig. 2(A)).

\section{BMI stratification}

When stratifying the study population in BMI classes (class I: $<18.5$; class II: $18.5-24.9$; class III: $25 \cdot 0-29.9$; class IV: $\geq 30.0 \mathrm{~kg} / \mathrm{m}^{2}$ ), we obtained significant vector displacement between class I and II, as well as between class III and class IV, whereas the vector migration between class II and III missed significance (see Fig. 2(B)). Phase angle was only significantly lower in class I than class II as well as III. As anticipated, $\mathrm{R} / \mathrm{H}$ decreased significantly with increasing BMI, whereas $\mathrm{Xc} / \mathrm{H}$ was only different between BMI class III and IV (see Table 2).
Anthropometric measurements such as arm muscle area and arm fat area decreased with decreasing BMI, age was only different between BMI class II and III/IV and sex distribution remained equal. Hand grip strength was significantly different between BMI class I and classes II-IV.

\section{Subgroup analysis}

We also aimed to study the vector migration caused by the SGA within the BMI classes, but only class II (18.5$24.9 \mathrm{~kg} / \mathrm{m}^{2}$ ) yielded sufficiently high patient numbers in the SGA classes (SGA A: $n$ 46; SGA B: $n$ 62; SGA C: $n$ 24) for comparative analysis. Here again, the SGA produced a distinguished vector migration, which was statistically significant between SGA A and B v. C. (see Fig. 3(A)) and revealed drastically altered Xc component with virtually unchanged resistance.

We also compared underweight by BMI (BMI class I $<18.5 \mathrm{~kg} / \mathrm{m}^{2}$ ) with malnutrition as assessed by the SGA (SGA C) and observed significant different vector and confidence ellipse positions (see Fig. 3(B)).

\section{Discussion}

In the present study we investigated how benign diseaserelated malnutrition determined by the SGA is reflected in the $\mathrm{R}$ Xc graph. We observed a significant vector migration dictated by the SGA, with a decreased Xc component implying abnormal tissue structure and integrity in malnutrition. These altered electric tissue properties were, however, not seen in patients with underweight according to BMI.

Sophisticated body composition in the routine clinical setting is difficult. BIA which is considered an easy non-invasive and inexpensive bed-side method is a reliable tool for body compartment calculation in healthy individuals, but equations for the calculation of body compartments must be validated for various disease states ${ }^{(6)}$. The BIVA providing information

Table 1. Demographic, nutritional and laboratory characteristics of the study population according to the Subjective Global Assessment (SGA) (Median values and interquartile ranges)

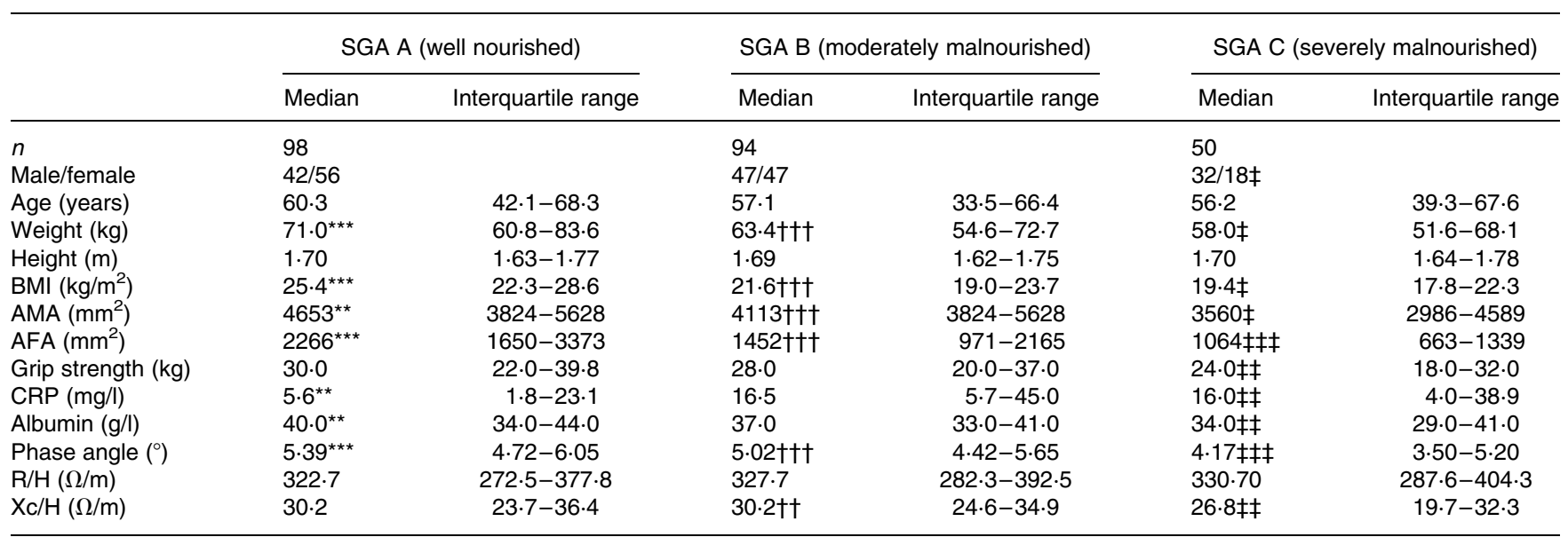

AFA, arm fat area; AMA, arm muscle area; CRP, C-reactive protein; $\mathrm{R} / \mathrm{H}$ : resistance standardized per height; $\mathrm{Xc} / \mathrm{H}$, reactance standardized per height.

Median values were significantly different from those of the SGA B group: ${ }^{\star \star} P<0.01,{ }^{\star \star \star} P<0.001$.

Median values were significantly different from those of the SGA C group: $\dagger \dagger P<0.01,1+\dagger P<0.001$.

Median values were significantly different from those of the SGA A group: $₫ P<0.05, \pm \pm P<0.01, \pm \pm \pm P<0.001$ 


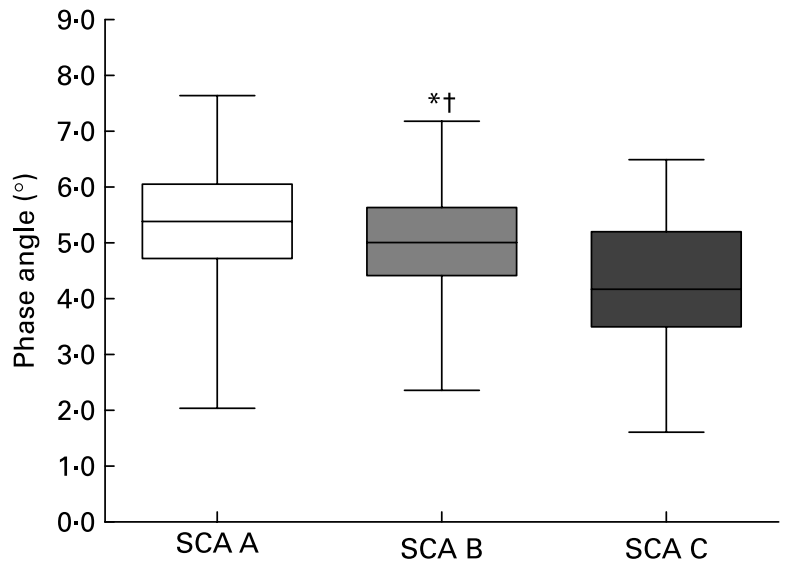

Fig. 1. Phase angle decreases significantly with Subjective Global Assessment (SGA). The box plots display the minimum, the maximum and the 25th, 50th and 75th percentiles. Values were significantly different from those of the SGA A group: ${ }^{*} P=0.033$. Values were significantly different from those of the SGA C group: $\dagger P<0.0001$.

on cell mass and integrity as well as hydration status has gained attention over recent years as it is independent from both body weight measurement or equation inherent errors $^{(10)}$.

In the present study population, the mean vector of the severely malnourished patients was significantly displaced when compared to both well-nourished and moderately malnourished patients. This vector migration was due to a significantly reduced Xc component with a preserved $\mathrm{R}$ component indicating impaired tissue structure in severe malnutrition. The $\mathrm{Xc} / \mathrm{H}$ and $\mathrm{R} / \mathrm{H}$ components of well-nourished and moderately malnourished patients were comparable.

There are several known factors influencing vector position and migration such as BMI, age, ethnic origin and sex. Increasing BMI is associated with both decreasing $\mathrm{R}$ and $\mathrm{Xc}$ components producing very typical vector patterns ${ }^{(17)}$.

Age and sex distribution was comparable between the SGA groups, but BMI decreased significantly with SGA class. It could therefore be expected that the vector migration would be entirely dictated/affected by the BMI thus indicating that the decreased Xc component in malnutrition is simply due to the lower body mass. When stratifying the study population according to BMI (class I: $<18.5$; class II: $18.5-24.9$; class III: 25.0-29.9; class IV: $\geq 30.0 \mathrm{~kg} / \mathrm{m}^{2}$ ), however, and studying the different vector patterns of the SGA classes and the BMI classes, it is apparent that vector displacement in severe malnutrition is not induced by the lower BMI. The mean vector of the BMI migrated in the opposite direction to the mean vector of the SGA when going from high BMI $\left(\geq 30 \mathrm{~kg} / \mathrm{m}^{2}\right)$ to low BMI $\left(<18.5 \mathrm{~kg} / \mathrm{m}^{2}\right)$.

The vector migration of the BMI groups is consistent with the vector migration shown in the retrospective analysis of the large NHANES databank by Piccoli et al. ${ }^{(18)}$ as well as the data by Bosy-Westphal et al. of 214294 healthy adults $^{(17)}$ with both $\mathrm{R} / \mathrm{H}$ and $\mathrm{Xc} / \mathrm{H}$ components actually decreasing with increasing BMI.

The pattern of the impedance vector distribution according to SGA classes, on the other hand, revealed virtually unaltered $\mathrm{R} / \mathrm{H}$ with significantly decreasing $\mathrm{Xc} / \mathrm{H}$ in the severely malnourished (SGA C), despite simultaneously decreased BMI.
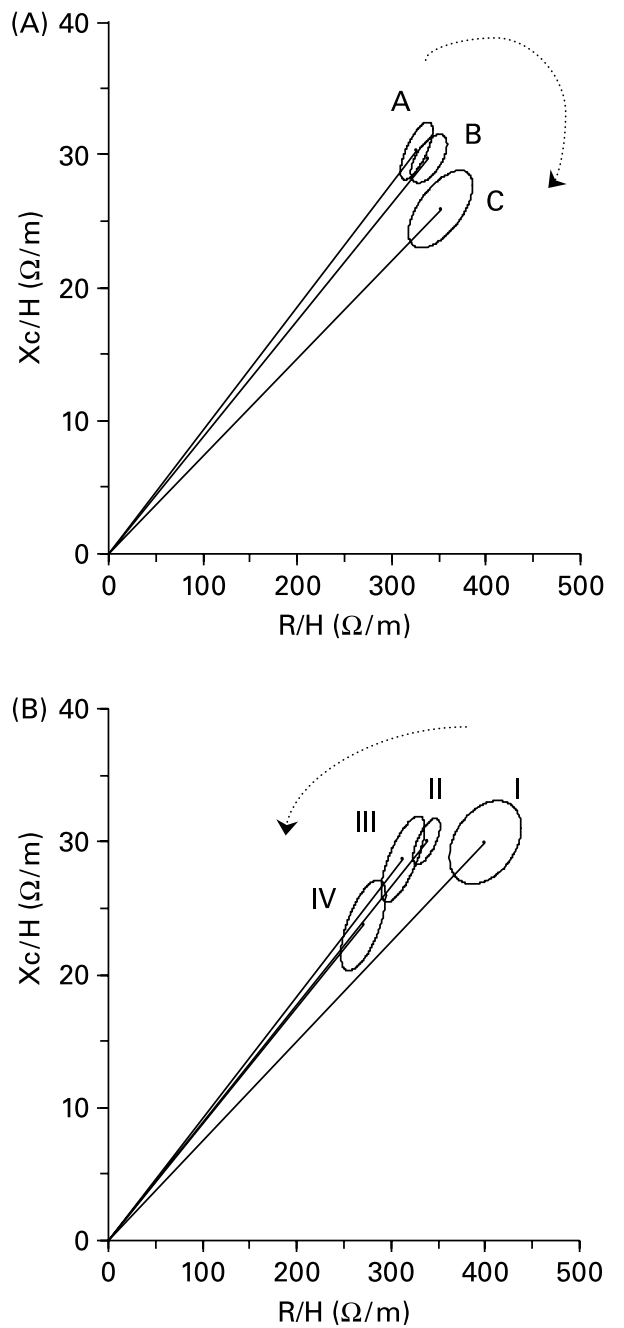

Fig. 2. (A), Significant vector migration $(\ldots \rightarrow)$ between well-nourished (Subjective Global Assessment (SGA) A) v. malnourished patients (SGA B and $C)$ due to decreased reactance standardized per height $(\mathrm{Xc} / \mathrm{H})$ component with preserved resistance standardized per height $(R / H)$. A v. B: $T^{2} 4.9, P=0.09, D$ 0.32; B v. C: $T^{2} 19.3, P<0.0001, D 0.77$. (B), Vector migration ( $\ldots .$. ) of BMI classes (I: $<18.5 \mathrm{~kg} / \mathrm{m}^{2}$; II: $18.5-24.9 \mathrm{~kg} / \mathrm{m}^{2}$; III: $\left.25-29.9 \mathrm{~kg} / \mathrm{m}^{2} ; \mathrm{IV}: \geq 30.0 \mathrm{~kg} / \mathrm{m}^{2}\right)$ in the opposite direction. I $v$. II: $T^{2} 33.4$, $P=0.0001, D$ 1.04; II v. III: $T^{2} 5.8, P=0.0575, D 0.42$; III v. IV: $T^{2} 9.4$ $P=0.0132, D 0.77$. See Subjects and methods section for details and explanation of confidence ellipses and vector migration. $D$, Mahalanobis distance; $T^{2}$, Hotelling's test.

Moreover, we obtained a similar vector migration dictated by the SGA in the subgroup analysis of patients with similar BMI $\left(18.5-24.9 \mathrm{~kg} / \mathrm{m}^{2}\right)$ which clearly indicates that the vector migration of the SGA is independent from BMI.

Also, comparing underweight patients with BMI $<18.5 \mathrm{~kg} / \mathrm{m}^{2}$ and patients who were classified severely malnourished according to the SGA (median BMI $19.4 \mathrm{~kg} / \mathrm{m}^{2}$ ), it is evident that these groups exhibit statistically different $\mathrm{R} / \mathrm{H}$ and $\mathrm{Xc} / \mathrm{H}$ values despite comparable BMI and phase angle.

The present results imply that malnutrition defined by the SGA is associated with abnormal tissue structure as well as loss of body mass and that these altered electric tissue properties are not seen in underweight according to BMI.

Disease-related malnutrition assessed by the SGA is mainly characterized by ongoing weight loss and is thus a marker of 
Table 2. Demographic, nutritional and laboratory characteristics of the study population according to BMI (Median values and interquartile ranges)

\begin{tabular}{|c|c|c|c|c|c|c|c|c|}
\hline & \multicolumn{2}{|c|}{ BMI class I $\left(<18.5 \mathrm{~kg} / \mathrm{m}^{2}\right)$} & \multicolumn{2}{|c|}{ BMI II $\left(18.5-24.9 \mathrm{~kg} / \mathrm{m}^{2}\right)$} & \multicolumn{2}{|c|}{ BMI III $\left(25-29.9 \mathrm{~kg} / \mathrm{m}^{2}\right)$} & \multicolumn{2}{|c|}{ BMI IV $\left(\geq 30.0 \mathrm{~kg} / \mathrm{m}^{2}\right)$} \\
\hline & Median & Interquartile range & Median & Interquartile range & Median & Interquartile range & Median & Interquartile range \\
\hline$n$ & 40 & & 132 & & 46 & & 24 & \\
\hline Male/female & $16 / 24$ & & $68 / 64$ & & $22 / 24$ & & $15 / 9$ & \\
\hline Age (years) & $45 \cdot 0$ & $28 \cdot 0-67 \cdot 0$ & 55.4 & $34 \cdot 1-65 \cdot 6$ & $66 \cdot 2 \dagger$ & $43 \cdot 3-70 \cdot 8$ & $65 \cdot 6$ & $58 \cdot 9-70 \cdot 0$ \\
\hline AMA $\left(\mathrm{mm}^{2}\right)$ & 3033 & $2619-3448$ & $4078^{\star \star \star}$ & $3533-4968$ & $5145+1 \dagger$ & $4379-5748$ & $6168 \ddagger \ddagger$ & $4828-6704$ \\
\hline AFA $\left(\mathrm{mm}^{2}\right)$ & 826 & $586-1083$ & $1587^{\star \star \star}$ & $1118-2193$ & 2701††† & $1881-3639$ & $3717 \ddagger$ & $2442-5173$ \\
\hline Grip strength (kg) & $24 \cdot 0$ & $18 \cdot 0-28 \cdot 0$ & $29 \cdot 5^{\star}$ & $20 \cdot 0-38 \cdot 3$ & $30 \cdot 0$ & $22 \cdot 5-36 \cdot 8$ & $29 \cdot 0$ & $22 \cdot 0-39 \cdot 8$ \\
\hline CRP $(\mathrm{mg} / \mathrm{l})$ & $18 \cdot 7$ & $3 \cdot 1-37 \cdot 0$ & $12 \cdot 8$ & $3 \cdot 2-35 \cdot 4$ & 11.6 & $3 \cdot 2-33 \cdot 6$ & $5 \cdot 0$ & $2 \cdot 6-11 \cdot 8$ \\
\hline Albumin $(g / l)$ & $36 \cdot 0$ & $29 \cdot 0-40 \cdot 0$ & $38 \cdot 0$ & $33 \cdot 0-43 \cdot 0$ & $40 \cdot 0$ & $34 \cdot 0-43 \cdot 0$ & $37 \cdot 0$ & $34 \cdot 0-43 \cdot 0$ \\
\hline Phase angle $\left({ }^{\circ}\right)$ & $4 \cdot 22$ & $3.49-5.41$ & $5 \cdot 07^{\star \star \star}$ & $4.44-5 \cdot 75$ & $5 \cdot 26$ & $4 \cdot 65-6 \cdot 13$ & $5 \cdot 17$ & $4 \cdot 66-5 \cdot 76$ \\
\hline $\mathrm{R} / \mathrm{H}(\Omega / \mathrm{m})$ & $396 \cdot 0$ & $327 \cdot 4-443 \cdot 6$ & $329 \cdot 0^{\star \star \star}$ & $284.5-390 \cdot 7$ & $315 \cdot 3 \dagger$ & $260 \cdot 1-366 \cdot 8$ & $266 \cdot 6 \ddagger \ddagger$ & $234 \cdot 3-300 \cdot 8$ \\
\hline $\mathrm{Xc} / \mathrm{H}(\Omega / \mathrm{m})$ & 31.4 & $24 \cdot 1-35 \cdot 3$ & 29.8 & $24.8-35.5$ & 29.9 & $21 \cdot 6-35 \cdot 3$ & $23 \cdot 1 \neq \ddagger$ & $18 \cdot 9-28 \cdot 6$ \\
\hline
\end{tabular}

AFA, arm fat area; AMA, arm muscle area; CRP, C-reactive protein; $\mathrm{R} / \mathrm{H}$ : resistance standardized per height; $\mathrm{Xc} / \mathrm{H}$, reactance standardized per height.

Median values were significantly different from those of the BMI class I group: ${ }^{\star} P<0.05$, ${ }^{\star \star \star} P<0.001$.

Median values were significantly different from those of the BMI class II group: $+P<0.05,+1+P<0.001$

Median values were significantly different from those of the BMI class III group: $\ddagger P<0 \cdot 05, \ddagger \ddagger P<0.01$.
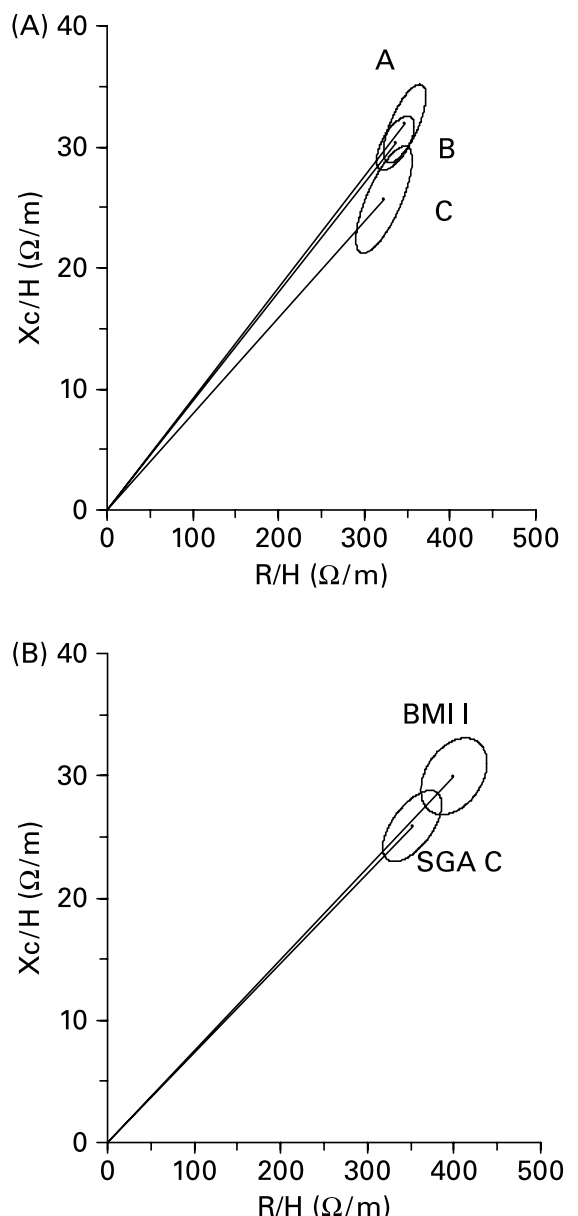

Fig. 3. (A), Significant vector migration between severely malnourished (Subjective Global Assessment (SGA) C) $v$. well- and moderately malnourished patients (SGA $A$ and $B$ ) due to decreased reactance standardized per height $(\mathrm{X} / \mathrm{H})$ component with preserved resistance standardized per height $(\mathrm{R} / \mathrm{H})$ within BMI class II $\left(18.5-24.9 \mathrm{~kg} / \mathrm{m}^{2}\right)$. A v. B: $T^{2} 1.2, P=0.554, D 0.21 ; \mathrm{B} v$. C: $T^{2} 8.7, P<0.017, D 0.71$. (B), Significantly displaced confidence ellipses and mean vectors of malnutrition (SGA C) $v$. underweight (BMI class I: $<18.5 \mathrm{~kg} / \mathrm{m}^{2}$ ). BMI I v. SGA C: $T^{2} 7.8, P=0.025, D 0.59$. See Subjects and methods section for details and explanation of confidence ellipses and vector migration. $D$, Mahalanobis distance; $T^{2}$, Hotelling's test. catabolism. It has repeatedly been shown that more than $10 \%$ weight loss in the preceding 6 months is invariably associated with clinically obvious impairment of organ function, and is predictive of postoperative complications ${ }^{(19,20)}$. The SGA does not consider current weight or BMI, which allows identification of malnourished patients despite a high or inconspicuous BMI.

Taken together, the present study, which is the first investigation of benign disease-related malnutrition in the BIVA, demonstrated significant disturbances in electric tissue properties in malnutrition as assessed by the SGA which are not seen in underweight according to BMI. The present results, moreover, add to the body of evidence that the BMI alone is not a suitable tool to identify disease-related malnutrition.

\section{Conclusion}

Clinical malnutrition as assessed by the SGA is associated with a distinctive bioelectrical vector migration which is strikingly different from the migration caused by the BMI. SGA vector migration is distinguishably marked by decreased Xc implying that abnormal tissue structure, and not reduced body mass only, occurs in disease-related malnutrition. These alterations are not seen in underweight according to BMI which is reflected by an increased R. BIVA appears to be an attractive tool to identify diseaserelated malnutrition and to monitor nutritional intervention.

\section{Acknowledgements}

This project was supported by a grant from the Charite Universitätsmedizin Berlin, research project no. 05/25369. K. N. and M. P. designed the study; C. S. and A. K. collected the data; K. N. analysed the data; L. V and H. L. provided significant advice; K. N., C. S., L. V. and M. P. wrote the manuscript. The authors have no conflicts of interest. 


\section{References}

1. Pirlich M, Schutz T, Norman K, et al. (2006) The German hospital malnutrition study. Clin Nutr 25, 563-572.

2. Norman K, Schutz T, Kemps M, Lubke JH, Lochs H \& Pirlich M (2005) The Subjective Global Assessment reliably identifies malnutrition-related muscle dysfunction. Clin Nutr 24, $143-150$.

3. Norman K, Kirchner H, Lochs H \& Pirlich M (2006) Malnutrition affects quality of life in gastroenterology patients. World J Gastroenterol 12, 3380-3385.

4. Barbosa-Silva MC, Barros AJ, Post CL, Waitzberg DL \& Heymsfield SB (2003) Can bioelectrical impedance analysis identify malnutrition in preoperative nutrition assessment? Nutrition 19, 422-426.

5. Barbosa-Silva MC \& Barros AJ (2005) Bioelectrical impedance analysis in clinical practice: a new perspective on its use beyond body composition equations. Curr Opin Clin Nutr Metab Care $\mathbf{8}, 311-317$.

6. Kyle UG, Piccoli A \& Pichard C (2003) Body composition measurements: interpretation finally made easy for clinical use. Curr Opin Clin Nutr Metab Care 6, 387-393.

7. Piccoli A (1998) Identification of operational clues to dry weight prescription in hemodialysis using bioimpedance vector analysis. The Italian Hemodialysis-Bioelectrical Impedance Analysis (HD-BIA) Study Group. Kidney Int 53, 1036-1043.

8. Piccoli A (2004) Bioelectric impedance vector distribution in peritoneal dialysis patients with different hydration status. Kidney Int 65, 1050-1063.

9. Coppini LZ, Waitzberg DL \& Campos AC (2005) Limitations and validation of bioelectrical impedance analysis in morbidly obese patients. Curr Opin Clin Nutr Metab Care 8, 329-332.

10. Piccoli A, Rossi B, Pillon L \& Bucciante G (1994) A new method for monitoring body fluid variation by bioimpedance analysis: the RXc graph. Kidney Int 46, 534-539.
11. Guglielmi FW, Mastronuzzi T, Pietrini L, Panarese A, Panella C \& Francavilla A (1999) The RXc graph in evaluating and monitoring fluid balance in patients with liver cirrhosis. Ann $N Y$ Acad Sci 873, 105-111.

12. Piccoli A, Pittoni G, Facco E, Favaro E \& Pillon L (2000) Relationship between central venous pressure and bioimpedance vector analysis in critically ill patients. Crit Care Med 28, 132-137.

13. Piccoli A, Brunani A, Savia G, Pillon L, Favaro E, Berselli ME \& Cavagnini F (1998) Discriminating between body fat and fluid changes in the obese adult using bioimpedance vector analysis. Int J Obes Relat Metab Disord 22, 97-104.

14. Detsky AS, McLaughlin JR, Baker JP, Johnston N, Whittaker S, Mendelson RA \& Jeejeebhoy KN (1987) What is subjective global assessment of nutritional status? JPEN $J$ Parenter Enteral Nutr 11, 8-13.

15. Gurney JM \& Jelliffe DB (1973) Arm anthropometry in nutritional assessment: nomogram for rapid calculation of muscle circumference and cross-sectional muscle and fat areas. Am J Clin Nutr 26, 912-915.

16. Piccoli A \& Pastori G (2002) BIVA Software. Padova: Department of Medical and Surgical Sciences, University of Padova.

17. Bosy-Westphal A, Danielzik S, Dorhofer RP, Later W, Wiese S \& Muller MJ (2005) Patterns of bioelectrical impedance vector distribution by body mass index and age: implications for bodycomposition analysis. Am J Clin Nutr 82, 60-68.

18. Piccoli A, Pillon L \& Dumler F (2002) Impedance vector distribution by sex, race, body mass index, and age in the United States: standard reference intervals as bivariate $\mathrm{Z}$ scores. Nutrition 18, 153-167.

19. Hill GL (1992) Jonathan E. Rhoads Lecture. Body composition research: implications for the practice of clinical nutrition. JPEN J Parenter Enteral Nutr 16, 197-218.

20. Windsor JA \& Hill GL (1988) Weight loss with physiologic impairment. A basic indicator of surgical risk. Ann Surg 207, 290-296. 